\title{
ROBUST DESIGN OF THE VIBRATORY GYROSCOPE WITH UNBALANCED INNER TORSION GIMBAL USING AXIOMATIC DESIGN
}

\author{
Kwang Hyeon Hwang \\ khhwang@ihanyang.ac.kr \\ Graduate Student \\ Department of Mechanical Design and Production \\ Engineering, Hanyang University \\ Gyung-Jin Park \\ gjpark@hanyang.ac.kr \\ Professor \\ Department of Mechanical Design and Production \\ Engineering, Hanyang University \\ Yong Chul Cho \\ yccho@sait.samsung.co.kr \\ Research Staff \\ MEMS Lab., Samsung Advanced Institute of \\ Technology
}

\begin{abstract}
Recently, there has been considerable interest in micro gyroscopes made of silicon chips. It can be applied to many micro-electro-mechanical systems (MEMS): devices for stabilization, general rate control, directional pointing, autopilot systems, and missile control. The mechanical design of the gyroscope is carried out using axiomatic design, followed by the application of the Taguchi robust design method to determine the dimensions of the parts so as to accommodate the dimensional variations during fabrication. Functional requirements are defined twofold. One is the natural frequencies should have fixed values, and the other is the system should be robust to large tolerances. According to the Independence Axiom, design parameters are classified into a few groups. Then, the detailed design process is performed according to the sequence indicated by the design matrix. The dimensions of the structure are determined to have constant values for the difference of frequencies regardless of the tolerances. It is noted that the Taguchi concept is utilized as a unit process of the entire axiomatic approach.
\end{abstract}

Keywords: Vibratory Gyroscope, Robust Design, Axiomatic Design, Decoupled Design

\section{INTRODUCTION}

The gyroscope is a device which measures the angular rates of an inertial object. It has been used in devices for stabilization, general rate control, directional pointing, autopilot systems, and missile control. Manufacturing of a mechanical gyroscope is very costly because various precision parts are required. Low-cost and small size gyroscopes are necessary for wider applications in the commercial field for accurate sensing.

In 1990, a company named Murata and Tokin produced a small vibratory gyroscope made of piezoelectric materials. Their

\author{
Kwon Hee Lee \\ leekh11@hanyang.ac.kr \\ Assistant Professor \\ Department of Mechanical Design and Manufacturing \\ Donga University \\ Byeong Leul Lee \\ bllee@saitgw.samsung.co.kr \\ Research Staff \\ MEMS Lab., Samsung Advanced Institute of \\ Technology \\ Seok Han Lee \\ 1sh@sait.samsung.co.kr \\ Vice President \\ MEMS Lab., Samsung Advanced Institute of \\ Technology
}

products were limited in size and costly because of the precision manufacturing process. Recently, there has been interest in the applications of silicon-made micro vibratory gyroscope because the size and cost could be considerably reduced [1-4].

The operating principle of a vertical gyroscope is based on the rotation-induced transfer of energy between two different vibration modes: driving mode and sensing (torsional) mode. If an angular velocity is imposed on the vibrating structure in the driving mode (horizontal direction) at the resonance frequency, it experiences Coriolis force. The Coriolis force proportional to the angular velocity causes displacements in the vertical direction. If the natural frequency in the sensing mode matches with the one in the driving mode, the displacements are exaggerated and sensing is easy [5]. Therefore, it is important to match the natural frequencies of two vibration modes [6,7]. However, lapping and etching processes make errors during fabrication. Fabrication error results in large dimensional error of the gyroscope. Such shape errors cannot be controlled easily. The dimensional error causes the variations in natural frequencies and thereby the low yield rate. To solve these problems, a robust design is applied to the design of a gyroscope. Han and Kwak performed robust optimal design considering fabrication errors [8]. In their approach, robust design was applied to a vibratory gyroscope without gimbal, and robustness is defined from optimization viewpoint.

This paper describes robust design of a vertical gyroscope with unbalanced inner gimbal. Axiomatic approach is chosen for the design process. The yield rate is increased although the fabrication errors exist. Evaluations of responses are conducted using a commercial FE program: GENESIS [9].

Functional requirements (FRs) of vibratory gyroscope are defined for robust design. Initial FRs of vibratory gyroscope are related to setting the frequencies of two vibration modes. In the conventional approach, an FR for robustness is not separately defined. FRs are defined for certain performances and robustness 
is achieved for a defined FR. In this research, it is noted that a separate FR is defined for robustness because robustness is not directly defined from existing FRs. The FR for robustness against fabrication errors is minimizing the variation of the difference of two natural frequencies to uncertain variables. According to the Independence Axiom [10,11], design parameters (DPs) are classified into groups for FRs. Design of Experiments (DOE) and Analysis of Variance (ANOVA) are used in this process. A design matrix is established by the sensitivity of a DP with respect to an FR. When the sensitivity is ignorable, the corresponding element in the design matrix is regarded as zero. As a result, a decoupled design is obtained through FRs-DPs mapping. Such a design has a sequence in solving procedure After making the design equation, each DP is determined to satisfy the corresponding FR. Robust design is conducted to satisfy the FR which is defined for robustness. Taguchi's concept is adopted to minimize the variance of the FR [12,13]. Dimensions of gyroscope are determined and an improved yield rate is achieved.

\section{REVIEW OF AXIOMATIC DESIGN AND TAGUCHI ROBUST DESIGN}

Axiomatic Design was proposed to provide a framework for design problems. It states that a design should be defined by independent FRs and DPs and designers should minimize the information content of their designs [10,11]. On the other hand, G. Taguchi had developed the Taguchi method for quality improvement [12]. Axiomatic Design and Taguchi method have found many successful applications in engineering and are continually being expanded to different design phases [14-18]. Taguchi method is utilized as a unit of the axiomatic approach in determining a DP to satisfy an FR.

\subsection{AXIOMATIC DESIGN}

The designers have to choose a correct set of DPs to be able to satisfy FRs. FR is "what we want to achieve" and DP is "how we achieve it." The world of design is made up of four domains: the customer domain, the functional domain, the physical domain, and the process domain $[10,11]$. The method is not explained in detail.

The basic postulate of the Axiomatic Design is that there are two fundamental axioms that govern the design process. The axioms are formally stated as:

Axiom 1: The Independence Axiom. Maintain the independence of functional requirements.

Axiom 2: The Information Axiom. Minimize the information content.

The Independence Axiom states that the independence of FRs must always be maintained, where FRs are defined as the minimum set of independent requirements that characterize the design goals. The relationship between the FR and the DP vectors are written as

$$
\{\mathbf{F R}\}=[\mathbf{A}]\{\mathbf{D P}\}
$$

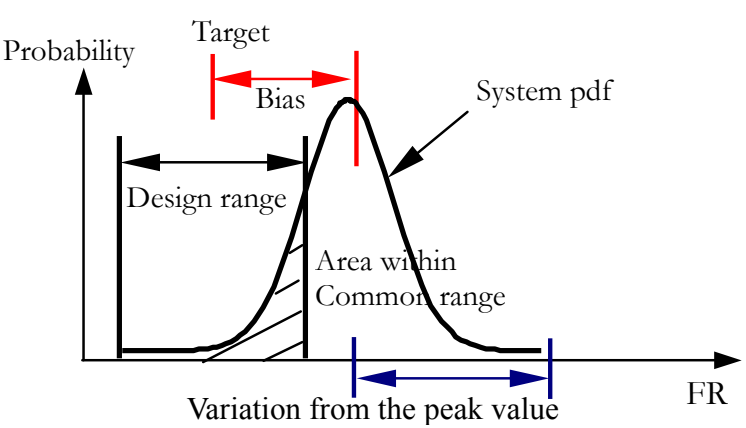

Figure 1. Design range, System range, Common range and System pdf for one FR

where $[\mathbf{A}]$ is called the design matrix. Based on the Independence Axiom, designs may be classified into three kinds: uncoupled, decoupled, and coupled. To satisfy the Independence Axiom, the design matrix must be either diagonal or triangular. When the design matrix is diagonal, an FR can be satisfied independently by one DP. Such a design is called an uncoupled design. When the matrix is triangular, the independence of FRs can be guaranteed if and only if the DPs are determined in a proper sequence. It is called a decoupled design. Any other form of the design matrix is called a full matrix and results in a coupled design.

The Information Axiom states that among those designs that satisfy the Independence Axiom, the design that has the smallest information content is the best design. The information content is defined in terms of the probability of success. A design that has the highest probability of success is the best design.

The design range and the system range of a design with one FR are illustrated in Figure 1. The probability of success is calculated as following:

$$
P_{s}=\frac{\text { Common range }}{\text { System range }}
$$

When an FR is a continuous random variable, the probability of success can be expressed as following:

$$
P_{s}=\int_{F R^{*}-\Delta}^{F R^{*}+\Delta} \phi(F R) d F R
$$

where $\phi(F R)$ is a system probability density function, $\Delta$ is a half of design range, and $F R^{*}$ is the target value.

\subsection{TAGUCHI ROBUST DESIGN}

Taguchi suggested that the steps for a product or a process design be composed of three kinds: system, parameter, and tolerance design. The system design is a step where new ideas are generated to provide products to customers. Within the parameter design step, the designer determines the optimum setting for control factors using orthogonal arrays and Signal-toNoise (SN) ratio. The final goal of the parameter design is that designer makes products insensitive to noise factors. The tolerance design is implemented to improve quality at a minimum cost. It should be used when the sensitivity of responses resulting 


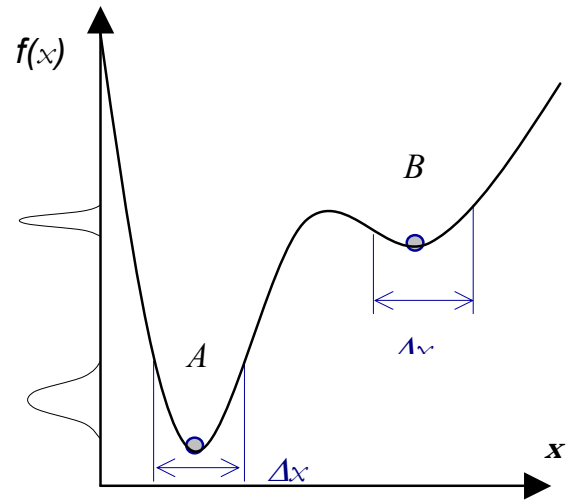

Figure 2. Robustness of $f(x)$

from the parameter design is not satisfactory. In particular, the parameter design scheme of the Taguchi method is adopted for robust design [16].

Figure 2 explains the concept of robust design with one control factor $x$. The objective function is $f(x)$ and the tolerance of the control factor is $\Delta x$. The distribution of the objective function is generated from that of the control factor. The tight dispersion of the objective function reduces the cost since the tolerance bands of the design variables can be loosened. In Figure 2, point A represents the optimum without considering the robustness, while point $\mathrm{B}$ represents the robust optimum. The performance of the deterministic optimum is better than that of the robust optimum. However, its distribution is wider than the robust optimum [17].

\section{STRUCTURE DESIGN AND FABRICATION PROCESS OF THE VIBRATORY VERTICAL GYROSCOPE}

The schematic of the vibratory vertical gyroscope is illustrated in Figure 3. The gyroscope is composed of four driving springs suspending the entire mass, the driving comb electrodes, and the driving-sensing comb electrodes. The bottom under the inner mass (gimbal) is an electrode that senses the tilting of inner mass. The inner mass and the outer frame are connected by two torsional springs. When the driving voltage applies on the driving comb electrode on the outer frame, the inner mass oscillates along $\mathrm{x}$-axis with the driving frequency. If the gyroscope rotates around $y$-axis, Coriolis force around $z$-axis is generated. The Coriolis force makes the asymmetry of the inner mass tilt, and the capacitance between the inner mass and the bottom electrode changes. The change is the sensing measure of the rotation.

In general, the surface micromaching using LPCVD (Low Pressure Chemical Vapor Deposition) poly silicon is used to fabricate the vibratory gyroscope with the bottom electrode. Figure 4 shows the micromaching fabrication process. When the structure layer is lapped and polished to $40 \mu \mathrm{m}$ thickness with CMP (Chemical Mechanical Polishing: see Figure 4.d), the variation of thickness occurs. When the gyroscope structure is formed with deep etcher (see Figure 4.e), the variation of width occurs. These variations cause the change of the frequency

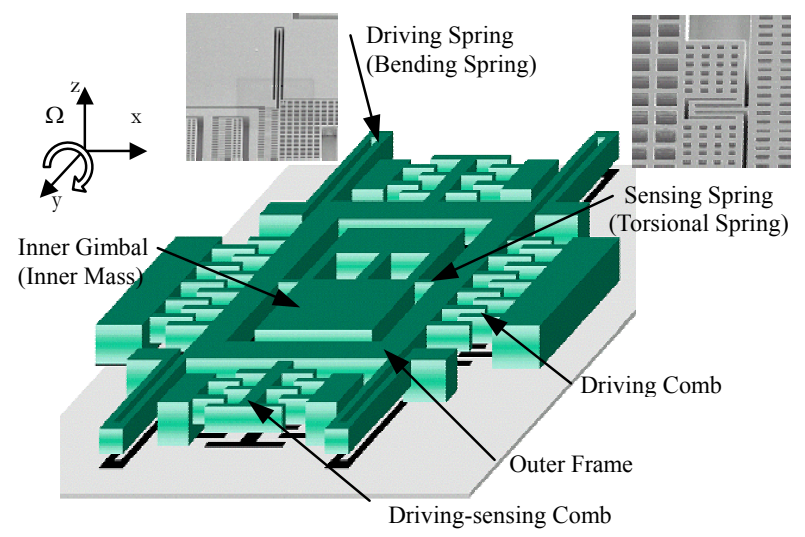

Figure 3. The schematic of the vibratory vertical gyroscope
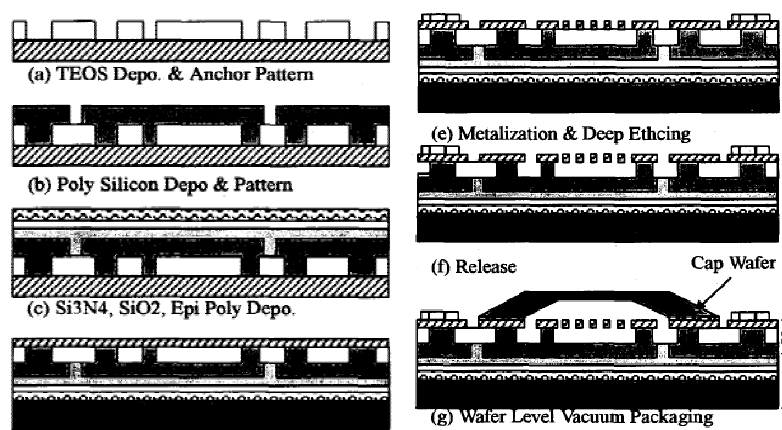

(d) SDB \& CMP

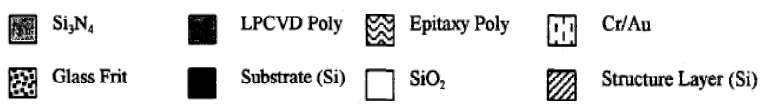

Figure 4. Manufacturing process

performance. Especially, driving frequency and sensing frequency have large changes so that the gyroscope does not operate well.

\section{ROBUST DESIGN OF GYROSCOPE}

The vibratory vertical gyroscope has two modes that are driving mode and sensing mode (see Figure 5). Two modes have to be perfectly separated by structural geometry and natural frequencies of two modes have to be very close each other. The gyroscope illustrated in Figure 3 has a possibility to satisfy these requirements. Driving mode is governed by bending springs and sensing mode is governed by torsional springs. However, the fabrication errors can change the desired dimensions of such springs. The change disturbs the separation of two modes and keeping the difference of two frequencies. As a result, the yield rate of products is reduced by the fabrication errors. To avoid the yield reduction, the difference of two frequencies has to be within specific range in spite of the fabrication errors.

The FRs and constraints of gyroscope with considering the fabrication tolerances are defined as follows:

FR1: Set the frequency $\left(f_{1}\right)$ of the driving mode.

FR2: Set the frequency $\left(f_{2}\right)$ of the sensing mode. 


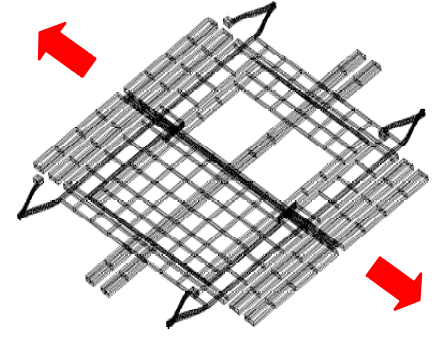

(a) Driving mode: 1 st frequency $\left(f_{1}\right)$

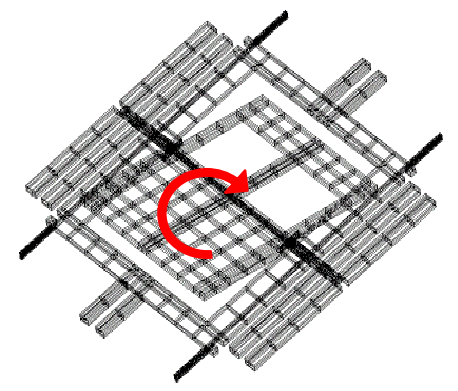

(b) Sensing mode: $2 n d$ frequency $\left(f_{2}\right)$

Figure 5. Driving mode and sensing mode of the vibratory vertical gyroscope

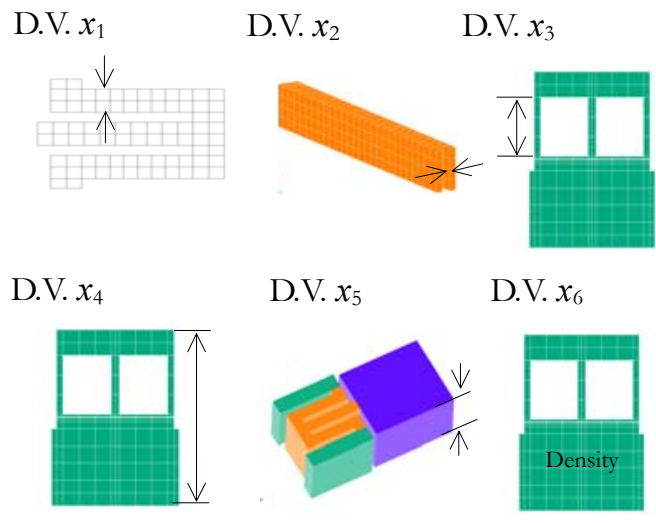

Figure 6. Design variables

FR3: Reduce the variation in the difference of two frequencies $\left(f_{2}-f_{1}\right)$.

Constraints:

C1: $f_{2}>f_{1}$

C2: $50 \mathrm{~Hz}<f_{2}-f_{1}<200 \mathrm{~Hz}$

Actually, C1 is a subset of C2. However, it is defined separately to emphasize the constraint.

FR1 and FR2 are related to the basic response in the operation of gyroscope. FR3 is defined to obtain the robustness. In the beginning of development, we thought that each variation of the natural frequencies have to be reduced. Therefore, only FR1 and FR2 were defined and the related DPs are determined to minimize the variations of them. When each variation was reduced, the difference of frequencies was increased. It was
Table 1. Levels of design variables for inner array $(\mu \mathrm{m})$

\begin{tabular}{ccccccc}
\hline Level & $\mathbf{x}_{\mathbf{1}}$ & $\mathbf{x}_{\mathbf{2}}$ & $\mathbf{x}_{\mathbf{3}}$ & $\mathbf{x}_{\mathbf{4}}$ & $\mathbf{x}_{\mathbf{5}}$ & $\mathbf{x}_{\mathbf{6}}$ \\
\hline 1 & $\mathrm{C}$ & $\mathrm{C}$ & $\mathrm{C}$ & $\mathrm{C}$ & $\mathrm{C}$ & $\mathrm{C}$ \\
2 & $\mathrm{C}-2$ & $\mathrm{C}-1$ & $\mathrm{C}+44.5$ & $\mathrm{C}-44.5$ & $\mathrm{C}+5$ & $\mathrm{C}-10 \%$ \\
3 & $\mathrm{C}+2$ & $\mathrm{C}+1$ & $\mathrm{C}-44.5$ & $\mathrm{C}-89$ & $\mathrm{C}+10$ & $\mathrm{C}-20 \%$ \\
\hline
\end{tabular}

Table 2. Levels of tolerances for outer array (\%)

\begin{tabular}{ccccccc}
\hline Level & $\mathbf{x}_{\mathbf{1}}$ & $\mathbf{x}_{\mathbf{2}}$ & $\mathbf{x}_{\mathbf{3}}$ & $\mathbf{x}_{\mathbf{4}}$ & $\mathbf{x}_{\mathbf{5}}$ & $\mathbf{x}_{\mathbf{6}}$ \\
\hline 1 & 0.00 & 0.00 & 0.00 & 0.00 & 2.50 & 0.00 \\
2 & -2.08 & -2.08 & -2.08 & -2.08 & 0.00 & 0.00 \\
3 & -4.17 & -4.17 & -4.17 & -4.17 & -2.50 & 0.00 \\
4 & -6.25 & -6.25 & -6.25 & -6.25 & -5.00 & 0.00 \\
5 & -8.33 & -8.33 & -8.33 & -8.33 & -7.50 & 0.00 \\
\hline
\end{tabular}

Table 3. $L_{25}$ all combination table

\begin{tabular}{ccccccc}
\hline No. & $\mathbf{x}_{\mathbf{1}}$ & $\mathbf{x}_{\mathbf{2}}$ & $\mathbf{x}_{\mathbf{3}}$ & $\mathbf{x}_{\mathbf{4}}$ & $\mathbf{x}_{\mathbf{5}}$ & $\mathbf{x}_{\mathbf{6}}$ \\
\hline 1 & 1 & 1 & 1 & 1 & 1 & 1 \\
2 & 1 & 1 & 1 & 1 & 2 & 1 \\
3 & 1 & 1 & 1 & 1 & 3 & 1 \\
4 & 1 & 1 & 1 & 1 & 4 & 1 \\
5 & 1 & 1 & 1 & 1 & 5 & 1 \\
6 & 2 & 2 & 2 & 2 & 1 & 2 \\
7 & 2 & 2 & 2 & 2 & 2 & 2 \\
8 & 2 & 2 & 2 & 2 & 3 & 2 \\
9 & 2 & 2 & 2 & 2 & 4 & 2 \\
10 & 2 & 2 & 2 & 2 & 5 & 2 \\
11 & 3 & 3 & 3 & 3 & 1 & 3 \\
12 & 3 & 3 & 3 & 3 & 2 & 3 \\
13 & 3 & 3 & 3 & 3 & 3 & 3 \\
14 & 3 & 3 & 3 & 3 & 4 & 3 \\
15 & 3 & 3 & 3 & 3 & 5 & 3 \\
16 & 4 & 4 & 4 & 4 & 1 & 4 \\
17 & 4 & 4 & 4 & 4 & 2 & 4 \\
18 & 4 & 4 & 4 & 4 & 3 & 4 \\
19 & 4 & 4 & 4 & 4 & 4 & 4 \\
20 & 4 & 4 & 4 & 4 & 5 & 4 \\
21 & 5 & 5 & 5 & 5 & 1 & 5 \\
22 & 5 & 5 & 5 & 5 & 2 & 5 \\
23 & 5 & 5 & 5 & 5 & 3 & 5 \\
24 & 5 & 5 & 5 & 5 & 4 & 5 \\
25 & 5 & 5 & 5 & 5 & 5 & 5 \\
\hline
\end{tabular}

found that each variation of two frequencies is not important. On the other hand, the sensitivity of bending springs should be similar to that of the torsional springs. Then the difference in FR3 will be robust. In general, achievement of robustness is not used as an FR. In this case, pursuing robustness is not directly related to FR1 and FR2. Therefore, a new FR for robustness is defined by FR3.

Figure 6 shows the potential design variables which can be changed in the design process. They are the torsional spring width $\left(x_{1}\right)$, the bending spring width $\left(x_{2}\right)$, the hole size of the gimbal $\left(x_{3}\right)$, the length of the gimbal $\left(x_{4}\right)$, the global thickness of the gyroscope $\left(x_{5}\right)$, and the density of the gimbal $\left(x_{6}\right)$. The global thickness of the gyroscope is fixed and only used to consider the fabrication tolerance. Actually, the gimblal is made of a porous 
Table 4. Sum of squres of each FR (ANOVA)

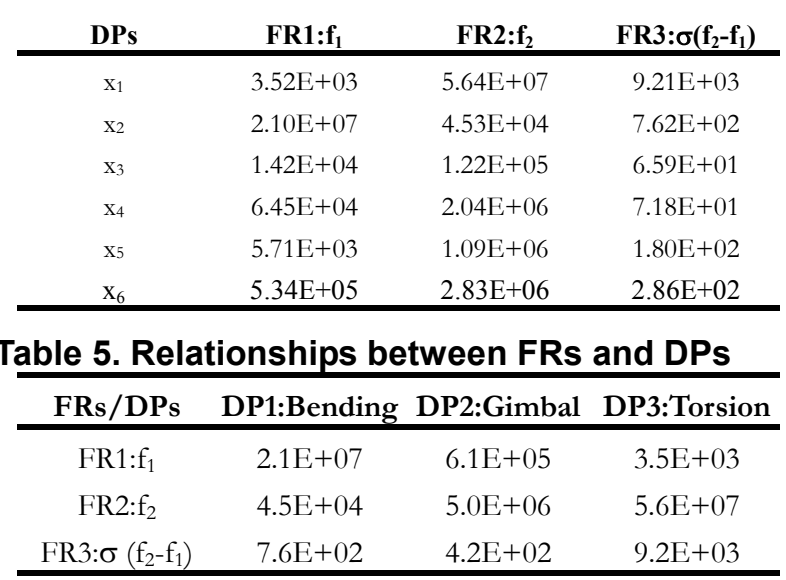

material which has etch holes. In finite element analysis, the density is utilized to represent the etch hole of the gimbal. The design variables are grouped to form DPs. The grouping is based on the sensitivity of the design variables with respect to FRs. The sensitivity can be evaluated from differentiation. However, differentiation only gives local information. Therefore, sensitivity in a region is employed, and it is calculated from the ANOVA table of Design of Experiment (DOE).

To evaluate the sensitivity, levels of design variables and five levels of tolerances are given in Tables 1 and 2, respectively. Orthogonal arrays are selected. The nominal values are located in the inner array and the tolerances are located in the outer array. The inner array uses the $\mathrm{L}_{18}$ standard array and the outer array uses $\mathrm{L}_{25}$ array (see Table 3 ) that has a full factorial combination. A finite element analysis is performed for each case of the inner and outer arrays. The total number of simulations is 450. ANOVA is performed from the results of simulations. The sum of squares is obtained from ANOVA as shown in Table 4. Finally, design variables are classified into three groups using the sum of squares. DP1 is the bending spring width $\left(x_{2}\right)$. DP2 has a set of design variables that are the hole size $\left(x_{3}\right)$, the length $\left(x_{4}\right)$ and the density $\left(x_{6}\right)$ of the gimbal. DP3 is the torsional spring width $\left(x_{1}\right)$. Each group is related to each FR and represents a DP. The relationship between FRs and DPs is shown in Table 5.

From the above analysis, the design equation is defined as follows:

$$
\left\{\begin{array}{l}
F R 1 \\
F R 2 \\
F R 3
\end{array}\right\}=\left[\begin{array}{ccc}
X & Y & z \\
z & Y & X \\
z & z & X
\end{array}\right]\left\{\begin{array}{l}
D P 1 \\
D P 2 \\
D P 3
\end{array}\right\}
$$

where $\mathrm{X}, \mathrm{Y}$ and $\mathrm{z}$ have the magnitude of $\mathrm{X}>\mathrm{Y}>\mathrm{z}$. The design can be considered as a decoupled design if $\mathrm{z}$ is assumed to be zero. Thus design has to be performed according to the sequence as indicated by equation (4). The first design activity is the adjustment of DP3, the second is DP2 and the third is DP1. Generally, it is difficult and costly to reduce variation after the design parameters are set to the mean response on the target. In order to keep the mean close to the target in product, the variation

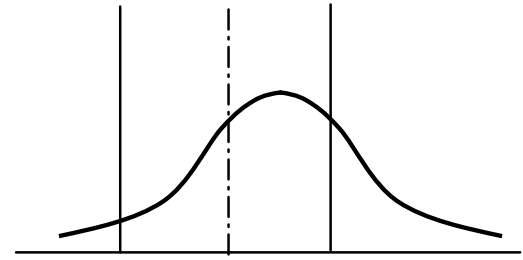

$\alpha L \quad \mu_{f_{2}-f_{1}} \quad \alpha u$

(a) Distribution of $f_{2}-f_{1}$ (before setting DP3)

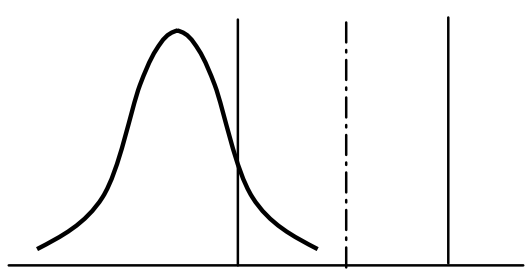

$\alpha L \quad \mu_{f_{2}-f_{1}} \quad \alpha U$

(b) Distribution of $f_{2}-f_{1}$ (after setting DP3)

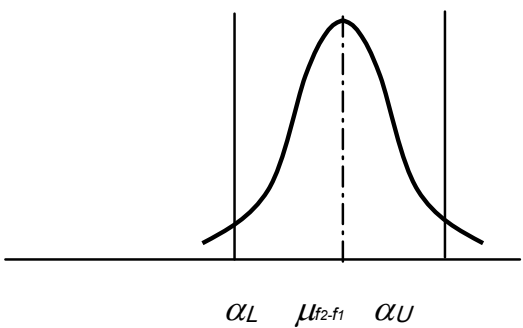

(c) Distribution of $f_{2}-f_{1}$ (after setting all DPs)

Figure 7. Changes of the system distribution

in the response must first be reduced [19]. The sequence in equation (4) matches the way in Taguchi method where system variation is reduced first and system target is controlled.

When we obtain the design equation, design process is very easy because the sequence for adjustment of DPs is known. The yield of gyroscope is represented by the distribution of the difference of two frequencies. Constraint C2 is a design range. At first, DP3 is determined to satisfy FR3. The tolerances of designs variables are assumed to have normal distributions. Although $x_{1}$ is only included in DP3, all the design variables are considered in the evaluation of the variance. The reason is that the off-diagonal terms in equation (4) are not exactly zeroes. The orthogonal array $\left(\mathrm{L}_{25}\right)$ in Table 3 is adopted for the calculation of the variance for FR3. The orthogonal array has a full factorial combination. The torsional spring width $\left(x_{1}\right)$ is only determined to reduce the variation of FR3 based on the result from $\mathrm{L}_{25}$. Figure 7.a illustrates the distribution of initial gyroscope design. After determining the DP3, the distribution is reduced as illustrated in Figure 7.b. It is noted that the variation is reduced but the mean value moves outside the design range. The next processes are utilized to adjust the response frequencies to the target values. The adjustment of nominal frequencies is a meaningless process because the nominal ones are changed by the fabrication errors. The changes of nominal values are due to the lack of symmetry in tolerances as shown in Table 1. Therefore, 


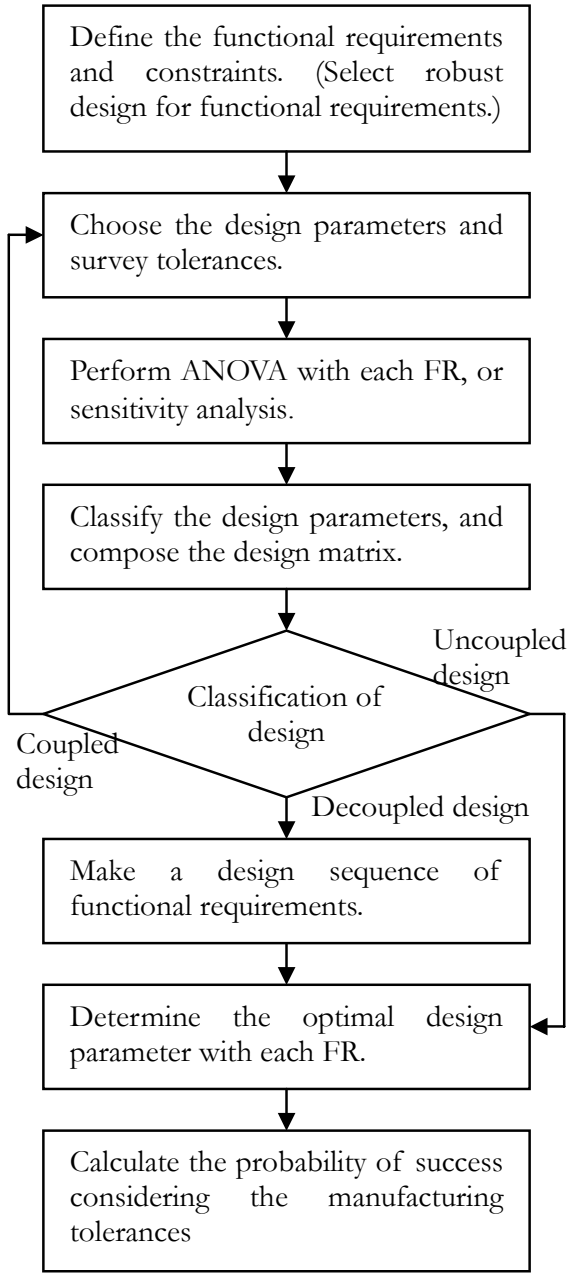

Figure 8. Robust design strategy

Table 6. Robust design results $(\mu \mathrm{m})$

\begin{tabular}{cc}
\hline Design variables & Values \\
\hline $\mathrm{x}_{1}$ & $\mathrm{C}-2$ \\
$\mathrm{x}_{2}$ & $\mathrm{C}-0.5$ \\
$\mathrm{x}_{3}$ & $\mathrm{C}+19$ \\
$\mathrm{x}_{4}$ & $\mathrm{C}-140$ \\
$\mathrm{x}_{5}$ & $\mathrm{C}$ \\
$\mathrm{x}_{6}$ & $\mathrm{C}-15 \%$ \\
\hline
\end{tabular}

C: Initial design value

mean values of frequencies are used instead of the nominal ones in the processes to determine DP2 and DP1. DP2 is determined to set the mean frequency to the target frequency without changing the variance of the distribution. The mean frequency in sensing mode is calculated by $\mathrm{L}_{25}$ array (see Table 3). DP2 is a set of design variables that is related to the gimbal. Actually, DP3 is also important in FR2. However, DP3 is fixed for FR2 to keep the variance of FR3. Therefore, we do not have much freedom in this process. Finally, DP1 is determined to satisfy FR1 by considering the fabrication errors of all design variables. The adjustment of the bending spring width $\left(x_{2}\right)$ is performed to adjust the frequency $\left(f_{1}\right)$ of the driving mode.

The final design distribution is shown in Figure 7.c. The variance is very small and the target values are kept in the design range. The flow of the design process is illustrated in Figure 8. The probability of success (Ps) is calculated by equation (3). Ps of the new design is increased by $15 \%$ compared to the initial design. Table 6 represents the final design. The springs become thin, and the gimbal shape is changed. This result shows that the yield of products is improved although the geometrical tolerances are allowed in fabrication.

\section{SUMMARY}

The robust design of the vibratory vertical gyroscope is conducted using the Axiomatic Design principle. The yield of gyroscope is increased by $15 \%$ compared to the initial design. The FRs and constraints are defined according to the objective of the design. Design variables are grouped for each FR. FRs-DPs mapping is carried out using sum of squares in ANOVA. To make the decoupled design, DOE and ANOVA apply to this process. An orthogonal array is adopted for the grouping process. Design equation is established to have a decoupled one. Design process is performed as indicated by the design equation.

\section{ACKNOWLEDGMENTS}

This research was supported by the Center of Innovative Design Optimization Technology, Korea Science and Engineering Foundation, and Samsung Advanced Institute of Technology (SAIT). The authors are thankful to Mrs. MiSun Park for her correction of the manuscript.

\section{REFERENCES}

[1] Tanaka, K., Mochida, Y., Sugimoto, S., Moriya, K., Hasegawa, T., Atsuchi, K. and Ohwada, K., "Micromachined Vibrating Gyroscope," Proc. MEMS'95, Amsterdam, Netherlands, pp. 278-281, 1995.

[2] Paoletti, F., Gretillat, M.-A. and de Rooij, N.F., "A Silicon Micromachined Vibrating Gyroscope with Piezoresistive Detection and Electromagnetic Excitation," Proc. MEMS'96, Atlanta, GA, pp. 162-167, 1996.

[3] Maenaka, K., Fujita, T., Konishi Y. and Maeda, M., "Analysis of a Highly Sensitive Silicon Gyroscope with Cantilever Beam as Vibrating Mass," Sensors and Actuators A: Physical, Vol. 54, pp. 568-573, 1996.

[4] Geiger, W., Folkmer, B., Sobe, U., Sandmaier, H. and Lang, W., "New Designs of Micromachined Vibrating Rate Gyroscope with Decoupled Oscillation Modes," Transducer'97, Chicago, IL, pp. 1129-1132, 1997.

[5] Lee, B.L., Lee, S.W., Jung, K.D., Choi, J.H. and Chung, T.R., "A De-coupled Vibratory Gyroscope using a Mixed Micromachining Technology," Proc. the 2001 IEEE Intern. Conference on Robotics \& Actuation, Vol. 4, pp. 3412-3416, 2001. 


\section{Robust Design of the Vibratory Gyroscope with Unbalanced Inner Torsion Gimbal Using Axiomatic Design Second International Conference on Axiomatic Design \\ Cambridge, MA - June 10-11, 2002}

[6] Mochida, Y., Tamura, M. and Ohwada, K., "A

Micromachined Vibrating Rate Gyroscope with Independent Beams for the Drive and Detection Modes," Micro Electro Mechanical Systems (MEMS 99), Twelfth IEEE Intern. Conference, pp. 618-623, 1999.

[7] Song, H., Oh, Y.S., Song, I.S., Choi, S.O., Kim, H.C., Ha, B.J., Baek, S.S. and Song, C.M., "Wafer Level Vacuum Packaged De-Coupled Vertical Gyroscope by Fabrication Process," Proc. Micro Electro Mechanical Systems (MEMS 2000), Thirteenth Annual Intern. Conference, pp. 520-524, 2000.

[8] Han, J. S., and Kwak, B. M., "Robust Optimal Design of a Vibratory Microgyroscope Considering Fabrication Errors," J. Micromech. Microeng. 11, pp. 662-671, 2001.

[9] Vanderplaatts, G.V., GENESIS Users Manual Ver. 6.0, VMA Engineering, 2000.

[10] Suh, N. P., The Principles of Design, The Oxford University Press, NewYork, 1990.

[11] Suh, N.P., Axiomatic Design: Advances and Applications, Oxford University Press, NewYork, 2001.

[12] Phadke, M.S., Quality Engineering Using Robust Design, Prentice Hall, New Jersey, 1989.

[13] Taguchi, G., Chowdhury, S. and Taguchi, S., Robust Engineering, McGraw-Hill, New York, 1999.

[14] Shin, M.K., Hong, S.W. and Park, G.J., "Axiomatic Design of the Motor-driven Tilt/Telescopic Steering System for Safety and Vibration," Proc instn Mech Engrs,Vol. 215, Part D, pp. 179-187, 2001.

[15] Hwang, Y.D. and Cha, S.W., "Axiomatic Design of Mold System for Advance of Foaming Magnitude," J. Korean Soc. Mech. Eng. A, Vol. 25, No. 4, pp. 637-644, 2001.

[16] Lee, K. H., Eom, I. S., Park, G. J. and Lee, W. I., "Robust Design for Unconstrained Optimization Problems Using the Taguchi Method," AIAA Journal, Vol. 34, No. 5, pp. 10591063, 1996.

[17] Lee, K.H. and Park, G.J., "Robust Optimization Considering Tolerance of Design Variables," Computers \& Structures, Vol. 79, No.1, pp. 77-86, 2001.

[18] Hwang, K.H., Lee, K.W. and Park, G. J., "Robust Optimization of An Automobile Rearview Mirror for Vibration Reduction," Structural and Multidisciplinary Optimization, Vol. 21, No. 4, pp. 300-308, 2001.

[19] Fowlkes, William Y. and Creveling, Clyde M., Engineering Methods for Robust Product Design, Addison-Wesley, Massachusetts, 1995. 